\title{
Geoestatística aplicada na geração de modelos numéricos de elevação
}

Carolina Ramalho Brito*

Carine Rezende Cardoso*

Alex da Silva Santos**

Resumo: Estudos de características do relevo, geomorfológicos, os quais abarcam amplas áreas do conhecimento, tais como agrimensura, cartografia, mineração, hidrologia, geologia, sensoriamento remoto, ciência do solo, ecologia e ciências ambientais, utilizam-se os modelos digitais de elevação (MDEs), baseado em processos numéricos essa é a técnica mais comum de derivação de atributos morfológicos de relevo em ambiente digital e tem contribuído na potencialização (rapidez e precisão) da obtenção de dados e informação. Diante disso, tem-se como objetivo avaliar se através do método de krigagem é possível melhorar a resolução espacial do SRTM referente a folha folha SF-23-X-B, que contempla a cidade e Viçosa dos valores de altitude, originalmente espaçados em 90 metros. Foram utilizados os softwares Quantum GIS 1.7, Microsoft巴 Office Excel 2007 e GS+ 7.0.

\section{Geostatistics applied to generate digital elevation model}

\begin{abstract}
Studies characteristics of relief, geomorphological, which cover broad areas of knowledge, such as surveying, mapping, mining, hydrology, geology, remote sensing, soil science, ecology and environmental sciences, we use the digital elevation models (DEMs), process-based numerical technique that is the most common derivation of morphological attributes relevant in the digital environment and has contributed to the enhancement (speed and accuracy) of obtaining data and information. Given this, one has to evaluate themselves through kriging method can improve the spatial resolution of the SRTM regarding leaf sheet SF-23-XB, which includes the city of Viçosa and altitude values, originally spaced at 90 meters. We used the software Quantum GIS 1.7, Microsoft ${ }^{\circledR}$ Office Excel 2007 and GS + 7.0.
\end{abstract}

Gérson Rodrigues dos Santos***

* Mestre em Agronomia pela Universidade Federal de Viçosa (UFV).

** Mestre em Engenharia Civil pela Universidade Federal de Viçosa (UFV).

*** Professor Adjunto da Universidade Federal de Viçosa (UFV).
Nilcilene das Graças Medeiros***

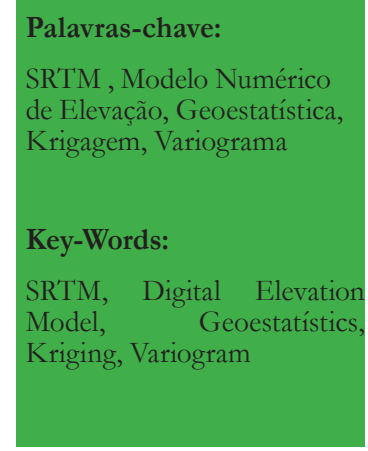




\section{Introdução}

Estudos de características do relevo, geomorfológicos, os quais abarcam amplas áreas do conhecimento, tais como agrimensura, cartografia, mineração, hidrologia, geologia, sensoriamento remoto, ciência do solo, ecologia e ciências ambientais, utilizam-se de modelos digitais de elevação (MDEs), baseado em processos numéricos (Heritage et al., 2009 apud Martins et al 2011), essa é a técnica mais comum de derivação de atributos morfológicos de relevo em ambiente digital e tem contribuído na potencialização (rapidez e precisão) da obtenção de dados e informação (Cherem, 2008).

A missão espacial da NASA (National Aeronautics and Space Administration), NIMA (National Imagery and Mapping Agency), DLR (Agência Espacial Alemã) e ASI (Agência Espacial Italiana) com a finalidade de gerar um modelo digital de elevação (MDE) da Terra usando a interferometria resultou no projeto SRTM (Shuttle Radar Topograpby Mission) (Torres, 2006). Dados SRTM tem sido bastante utilizados para a representação digital do relevo.

A resolução dos dados SRTM é de $90 \mathrm{~m}$, o que parece ser razoável, no entanto em estudos onde requer maior precisão, o mesmo torna-se inadequado. Uma alternativa para se obter uma superfície com coerência, consiste em interporlar os dados originais com uma resolução maior (Grohmann et al., 2008).

Segundo Valeriano (2008), a krigagem, uma técnica geoestatística, é o método de interpolação que melhor expressa as formas do relevo. Esse método permite a interpolação de valores de variáveis não amostradas a partir dos vizinhos amostrados. A cota de um ponto de interesse é calculada pela média ponderada das amostras vizinhas, determinada por meio de análise geoestatística, a qual fornecerá os coeficientes que descrevem, em semivariogramas, a variabilidade espacial dos dados analisados. (Valeriano, 2008).

Atualmente há um crescimento significativo no emprego da geoestatística e sua aplicação em distintas áreas do conhecimento como na geografia, ecologia, na climatologia, na engenharia, inclusive sua implementação em Sistemas de Informação Geográficas (SIG).

Valeriano (2002, 2003, 2004, 2008) e Valeriano e Abdon (2007) fizeram uso da geoestatística para a extração de informações do relevo através de Modelos Digitais de Elevação (MDE), que são representações digitais de uma variação contínua do relevo no espaço (Burrough, 1986; Almeida e Berger, 2007).

Diante disso, tem-se como objetivo avaliar a possibilidade de melhorar a resolução espacial do SRTM através do método de krigagem.

\section{Materiais e métodos}

Inicialmente foi definido um retângulo delimitando o município de Viçosa

Geografia Ensino \& Pesquisa, v. 17, n.3, p. $143-152$, set./dez. 2013

Geoestatística aplicada na geração de modelos numéricos de elevação (Figura 1), cuja a imagem é referente a folha SF-23-X-B, que contempla a cidade, com valores de altitude, originalmente espaçados em 90 metros, a fim de analisar parcialmente o SRTM, através dos métodos geoestatísticos.

O procedimento acima foi realizado em ambiente Quantum GIS 1.7, através da extensão GDAL (Geospatial Data Abstraction Library). A partir do recorte foi gerado um arquivo com a extensão XYZ, que contempla os pares de coordenadas geográficas e altimetria em metros presentes na imagem SRTM, com referencial geodésico WGS-84. 
Estes dados foram tratados no Microsoft ${ }^{\circledR}$ Office Excel 2007 e importados para a plataforma do GS +7.0 , onde a análise geoestatística foi realizada, para definir o melhor ajuste ao variograma.

A Figura 2 apresenta o histograma dos dados observados de altimetria na região de Viçosa.

Figura 1 - Área de Interesse (AOI), retângulo envolvente do município de Viçosa.

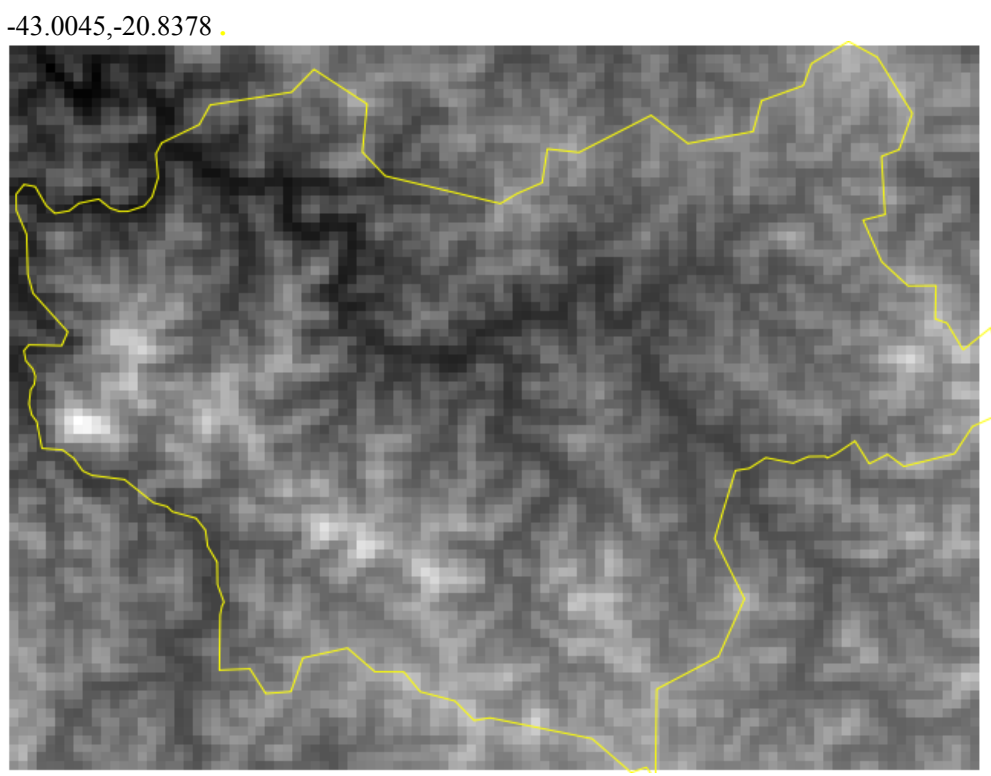

Fonte: elaborado pelos autores.

Figura 2 - Histograma do recorte do SRTM Viçosa.

Histograma SRTM Viçosa

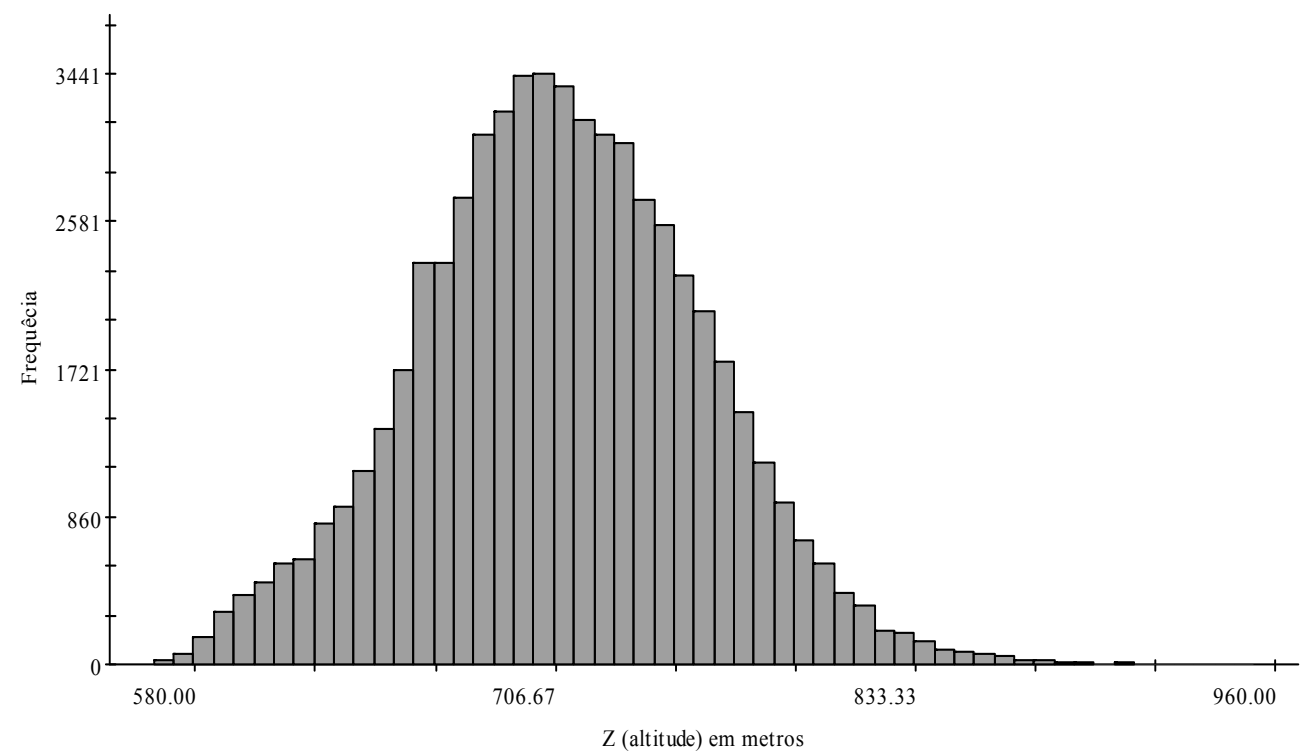

Fonte: elaborado pelos autores.

Geografia Ensino \& Pesquisa, v. 17, n.3 p. 143-152, set./dez. 2013.

Brito, C. R.; Cardoso, C. R.; Santos, A.S.; Santos, G. R.; Medeiros, N. G. 


\section{Resultados e discussão}

A seguir são apresentados procedimentos para definir os parâmetros a serem considerados na técnica de krigagem.

\section{Análise descritiva dos dados}

A Tabela 1 ilustra o resumo estatísticos dos valores altimétricos observados.

Tabela 1 - Análise descritiva dos dados altimétricos.

\begin{tabular}{c|c|c|c|c}
\hline $\begin{array}{c}\text { Número de } \\
\text { observaçóes(n) }\end{array}$ & Módia & Variância & $\begin{array}{c}\text { Deswio } \\
\text { padtão }\end{array}$ & Outiliers \\
\hline 59748 & 719.707 & 2494.819 & 49.948 & - \\
\hline
\end{tabular}

Fonte: elaborado pelos autores.

\section{Análise de tendência}

A Anisotropia ocorre quando o variograma apresenta valores diferentes do Efeito Pepita (C0), e da Contribuição (C) e/ou o modelo é diferente ao mudar a direção de h. A anisotropia quando ocorre o mesmo Patamar $(\mathrm{C} 0+\mathrm{C}$ ), mas alcances (a) diferentes do mesmo modelo (Figura 3).

Figura 3 - Representação gráfica de semivariogramas anisotrópicos.

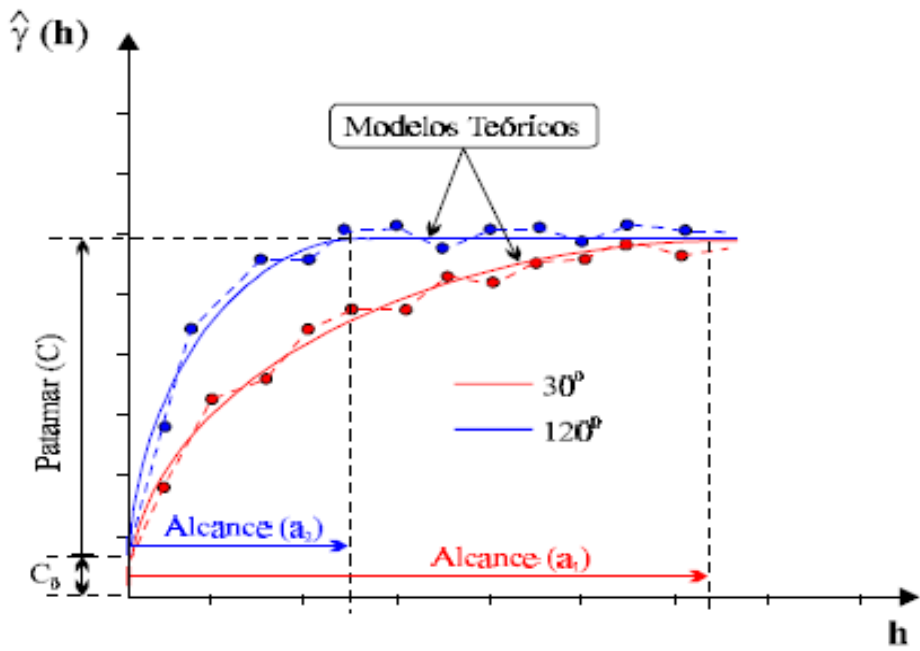

Fonte: CAMARGO, 2011.

A anisotropia é classificada em 3 (três) tipos:

- Geométrica - é a razão entre o alcance na direção de menor continuidade (a2) e o alcance na direção de maior continuidade (a1). Neste caso, o valor é menor que a unidade e o ângulo é o mesmo da direção de máxima continuidade.

- Zonal - quando apresenta o mesmo alcance e diferentes patamares.

Geografia Ensino \& Pesquisa, v. 17, n.3, p. $143-152$, set./dez. 2013

Geoestatística aplicada na geração de modelos numéricos de elevação

- Combinada - é a situação mais comum, onde existe característica da anisotropia zonal e geométrica, denominada como combinada.

Isotropia - quando o variograma é idêntico em todas as direções. A figura a seguir ilustra uma situção de isotropia.

Analisando o semivariograma nas quatro direções, foi observado uma leve tendência a anisotropia geométrica dos dados, conforme Figura 4. 
Figura 4 - Análise de tendência.
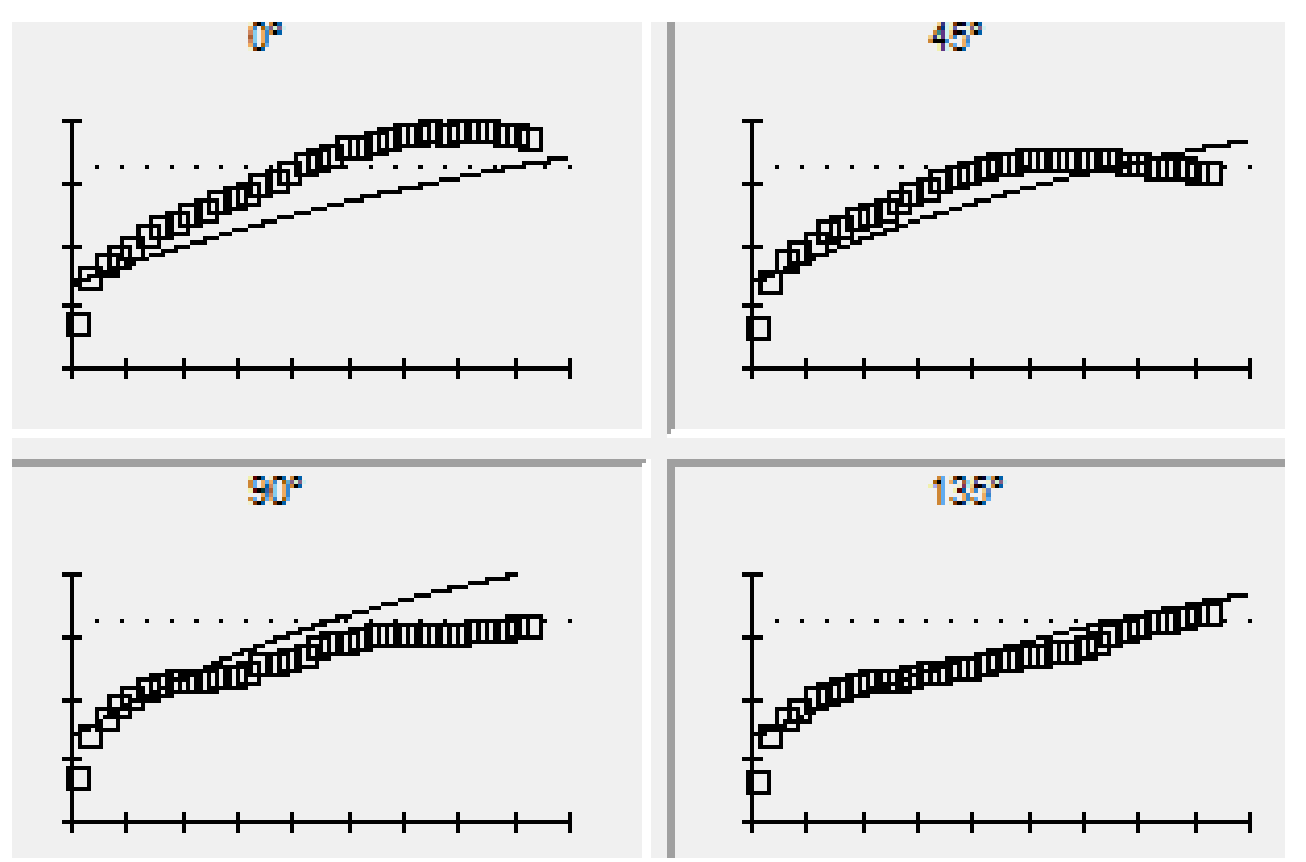

Fonte: elaborado pelos autores.

\section{Ajuste do variograma}

A Tabela 2 apresenta os parâmetros identificados para o melhor ajuste do variograma.

Tabela 2 - Parâmetros para os dados altimétricos do SRTM.

\begin{tabular}{c|c|c|c|c|c|c|c}
\hline $\begin{array}{c}\text { Modelo } \\
\text { Teónico }\end{array}$ & $\begin{array}{c}\text { Distância } \\
\text { (d) }\end{array}$ & $\mathrm{C}_{0}$ & $\mathrm{C}_{0}+\mathrm{C}$ & $\mathrm{A}_{0}$ & $\mathrm{RSS}$ & $\mathrm{R}^{2}$ & IDE (\%) \\
\hline Exponencial & Olograus & 680 & 2625 & 0.03 & 199897 & 97.6 & 0.741 \\
\hline
\end{tabular}

Fonte: elaborado pelos autores.

Onde:

Alcance (a) - é a distância na qual os valores observados ainda apresentam dependência espacial.

Patamar (C) - é o valor do semivariograma correspondente ao alcance.

Efeito Pepita (CO) - a medida que a distância (h) tende a 0 (zero) o semivariograma aproximase de um valor positivo, denominado Efeito Pepita, revelando sua descontinuidadade para distâncias menores que a menor distância entre as amostras.

Contribuição (C1) - é a diferença entre o Patamar (C) e o Efeito Pepita (C0)

Erro Quadrático Padrão (RSS)

IDE (Índice de Dependência Espacial): índice calculado pela relação [C / $(\mathrm{C} 0+\mathrm{C})]$, conforme critérios estabelecidos por Zimback (2001), onde assume-se os seguintes intervalos:

$\mathrm{IDE} \leq 25 \%-$ Fraco

Geografia Ensino \& Pesquisa, v. 17, n.3 p. $143-152$, set./dez. 2013.

$25 \%<\mathrm{IDE}>75 \%$ - Moderado

Brito, C. R.; Cardoso, C. R.; Santos, A. S.; Santos, G. R.; Medeiros, N. G.

IDE $>75 \%$ - Forte 
A Figura 5 apresenta o variograma ajustado.

Figura 5 - Variograma ajustado.

\section{Z: Variograma Isotropico}

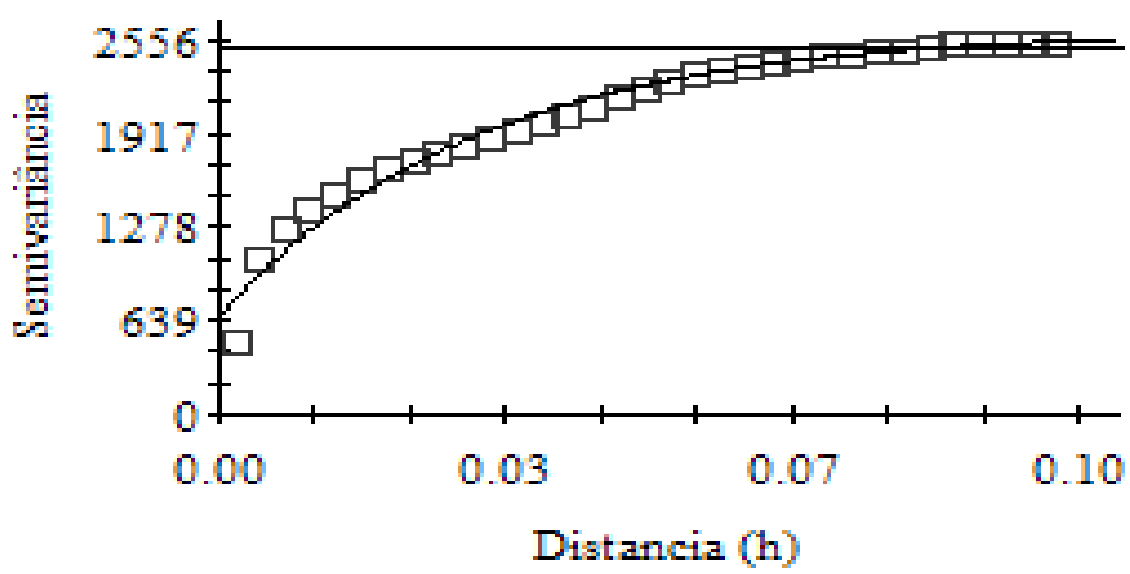

Fonte: elaborado pelos autores.

Uma das ferramentas principais da geoestatística é a função chamada de variograma (ou semivariograma), que basicamente descreve a variabilidade espacial do processo estocástico gerador das observações da variável regionalizada de interesse (Mingoti, 1995). O variograma é, na verdade, uma medida de distância entre as observações no espaço e na prática é estimado através dos dados amostrais coletados na região (espaço) de investigação.

$\mathrm{Na}$ análise espacial de dados via geoestatística, existem duas fases na estimação do variograma. A primeira consiste em obter-se o variograma experimental do processo, também chamado de variograma amostral. Os estimadores mais comuns nesta fase são: o clássico de Matheron (1963), o robusto proposto por Cressie e Hawkins (1980) e o das medianas (Cressie,1993).

Através do cálculo do variograma experimental, podemos reconhecer qual a forma matemática da função de variabilidade espacial do processo estocástico gerador dos dados amostrais, o qual é chamado de variograma teórico do processo e então, estimar seus parâmetros, o que constitui a terceira fase.

O método de estimação de parâmetros mais usado é o de Mínimos Quadrados Ponderados proposto por Cressie (1985), apesar de existirem outros procedimentos como o de Mínimos Quadrados Generalizados e Máxima Verossimilhança, entre outros (Cressie, 1993).

\section{Auto-validação}

Os modelos foram testados pelo processo de "autovalidação", para definir

Geografia Ensino \& Pesquisa, v. 17, n.3, p. $143-152$, set./dez. 2013

Geoestatística aplicada na geração de modelos numéricos de elevação qual o melhor modelo de interpolação. Neste momento é levado em consideração os valores de $\beta 0$ (próximo a 0) , $\beta 1$, r2 (próximo a 1) e Erro Padrão (SE) ((próximo a 0), presentes na Tabela 3. 
Tabela 3 - Parâmetros observados na auto-validação.

\begin{tabular}{c|c|c|c}
\hline$\beta_{0}$ & $\beta_{1}$ & $\mathrm{r}^{2}$ & $\mathrm{SE}$ \\
\hline 1.073 & -52.65 & 0.939 & 0.001 \\
\hline
\end{tabular}

Fonte: elaborado pelos autores.

Onde:

SE - Erro padrão ou Erro Reduzido

$\beta 0$ - coeficiente linear

$\beta 1$ - coeficiente angular

$\mathrm{r}^{2}$ - coeficiente de ajuste

Figura 6 - Validação cruzada

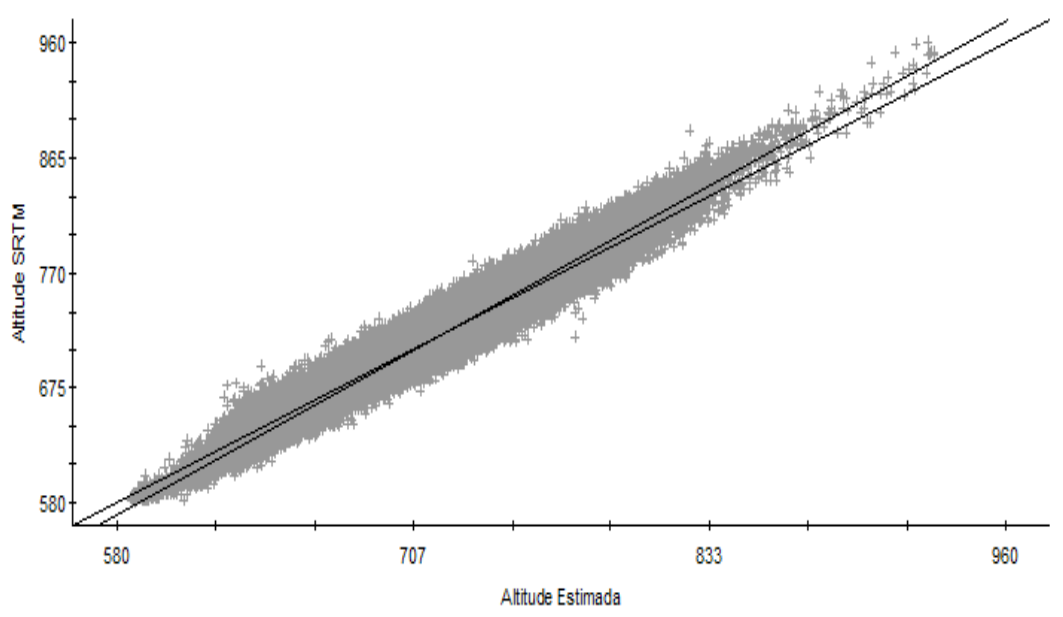

Fonte: elaborado pelos autores.

\section{Krigagem}

Os tipos de krigagens comumente usados são a krigagem ordinária e a krigagem universal, cujas equações são apresentadas, as seguir (Druck et.al., 2004):

Krigagem ordinária

$$
\hat{Z}(\mathrm{x})=\sum_{i=1}^{n} \lambda_{i} Z\left(x_{i}\right)
$$

Krigagem universal

$$
\text { [2] } \hat{Z}(\mathrm{x})=\mathrm{t}(\mathrm{x})+\sum_{i=1}^{n} \lambda_{i} Z\left(x_{i}\right)
$$

Onde:

Z (x) é o valor estimado para uma dada posição;

Geografia Ensino \& Pesquisa, v. 17, n.3 p. 143-152, set./dez. 2013

$\lambda \mathrm{i}$ (Zi) são ponderações que estabelecem a contribuição com que cada observação de Z contribui para a estimativa de Z (x);

Brito, C. R.; Cardoso, C. R.; Santos, A. S.; Santos, G. R.; Medeiros, N. G.

t (x) é uma componente de tendência. 
Figura 7 - Mapa de isolinhas gerado.

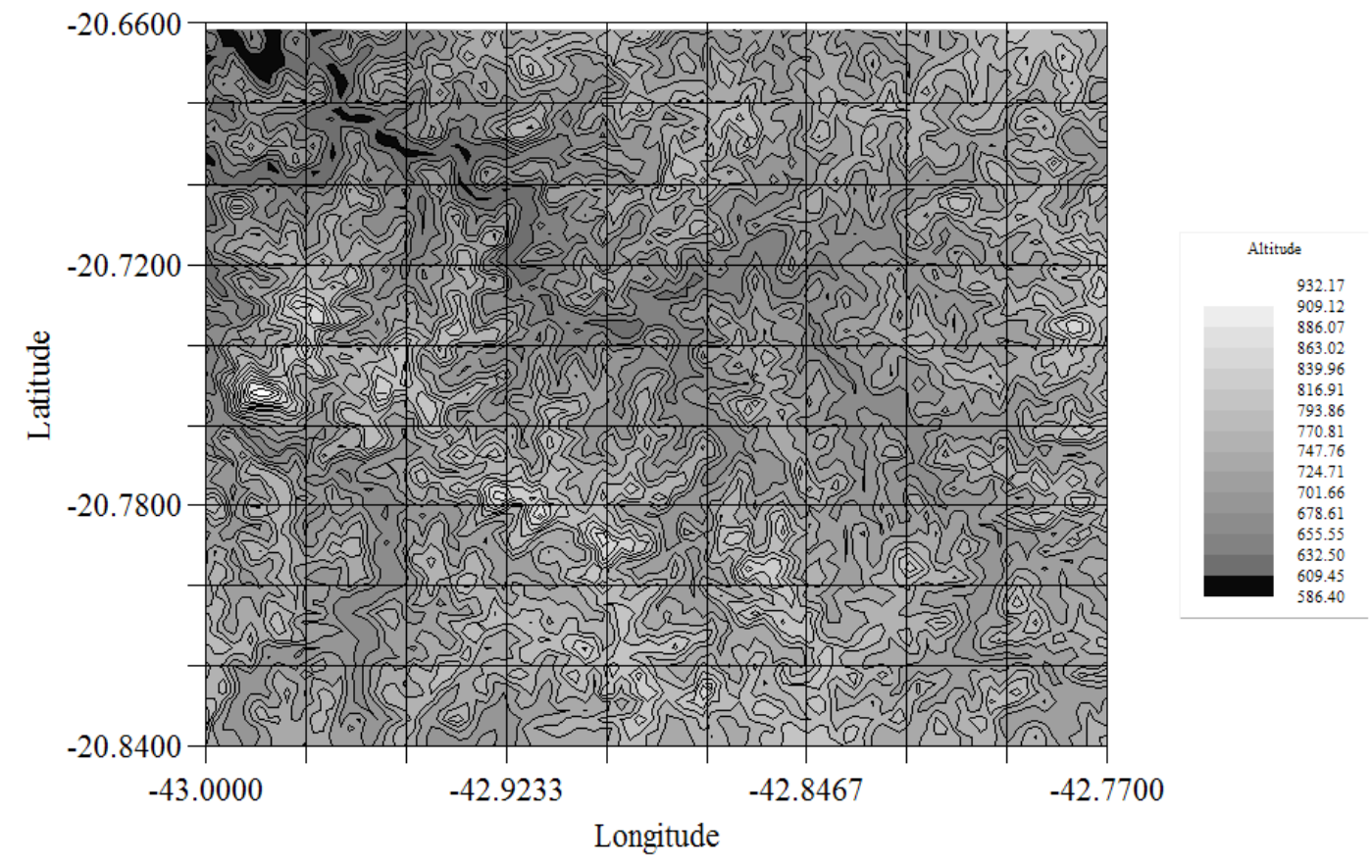

Fonte: elaborado pelos autores.

Incertezas

Em estudo, a princípio o GS+ não gerou o mapa de incertezas.

\section{Considerações gerais}

No mapa de isolinhas gerado é possível identificar as características morfológicas do relevo do município de Viçosa, assim como a análise de sua rede de drenagem.

Pela auto-validação foi possível observar o erro entre os valores estimados e os presentes na imagem SRTM.

Em estudo a comparação dos valores estimados pela krigagem com dados coletados em campo.

\section{Referências}

ALMEIDA, A. Q. de.; BERGER, B. Comparação entre áreas de preservação permanente demarcadas a partir de diferentes escalas topográficas. In: MORAIS, A. C. de.; SANTOS, A. R. dos. (Org.).

Geografia Ensino \& Pesquisa, v. 17, n.3, p. $143-152$, set./dez. 2013

Geoestatística aplicada na geração de modelos numéricos de elevação
Geomática e análise ambiental: aplicações práticas. Vitória: EDUFES, 2007. cap. 1, p.19-39. ANTUNES, F.Z. 1979. Contribuição para a caracterização do regime hídrico de Minas Gerais e aptidão das principais culturas. Inf.Agropec. 5: 43-78.

CAMARGO, E. C. G. Geoestatística: fundamentos e aplicações. [S. 1.: s. n.], 1998. cap. 5. Disponível em: < http://www.dpi.inpe.br/gilberto/tutoriais/gis_ambiente/>. Acesso em: 20 de out. de 2011. 
CRESSIE, N. Statistics for spatial data. New York: John Wiley \& Sons, 1993.

CRESSIE, N.; HAWKINS, M. Robust estimation of the variogram: I. Mathematical Geology, 12 (2): 115-125,1980.

CRESSIE, N. Fitting variogram models by weighted least squares, Mathematical Geology, 17, 5, 563$582,1985$.

FERNANDES, L. S. Avaliação de mantas comerciais na vegetação de talude em corte de estrada. Dissertação de Mestrado. Viçosa: UFV, 2004. p.81.

FREITAS, V. A. de. Análise de dados espaciais por meio de semivariogramas. Uberlândia: Universidade Federal de Uberlândia, 2000. 27 p.

HUIJBREGTS, C. J. Regionalized variables and quantitative analysis of spatial data. In: Davis, J.C. E McCullagh, M.J. (ed) Display and anaysis of spatial data. New York, John Wiley. p.38-53, 1975.

ISAAKS, E. H.; SRIVASTAVA, M. An introduction to applied geostatistics. New York: Oxford University Press, 1989.600 p.

KER, João Carlos. Anotações e perguntas sobre solos brasileiros (SiBCS-atualizado). Viçosa: UFV, 2006.

MATHERON, G. Principles of geostatistics. Economic Geololy, 58,1246-1266,1963.

MINGOTI, S. A., SILVA, A. F. Um exemplo de aplicação das técnicas de Geoestatística: Uma análise do nível piezométrico do aquífero de Wolfcamp (Estados Unidos). Revista Escola de Minas (REM), 49, 02, 96-100, 1995.

MINGOTI, S., A., NEVES, F., O., A metodologia de geoestatística como alternativa na análise de séries temporais. Revista Escola de Minas (REM), 52, 03, 182-187, 1999.

NIMER, E. 1977. Clima. In: Geografia do Brasil. Região Sudeste (C. Goldenberg, ed.). IBGE, Rio de Janeiro, p.51-89.

ROSA, G. Avaliando a Qualidade dos Estimadores de Variograma (Variograma Experimental) e do Método de Mínimos Quadrados Ponderados para Estimação dos Parâmetros do Modelo de Variograma Teórico do Processo. Dissertação de Mestrado, 2003.

TONELLO, K. C.; Dias, H. C. T.; Souza, A. L.; Alvares, C. A.; Ribeiro, S.; Leite, F. P. Morfometria da Bacia Hidrográfica da Cachoeira das Pombas, Guanhães - MG. Revista Árvore, v.30, n.5, p.849-857, 2006.

TORRES, T.G.; FILHO, A.C.P.; JUNIOR, H.T.; CORREA, L.C.; GARCEZ, A.J.S.; COPATTI, A. Utilização dos dados SRTM na geração dos limites da bacia hidrográfica do rio Formoso (Bonito, MS). Anais... $1^{\circ}$ Simpósio de Geotecnologias no Pantanal, Campo Grande, Brasil, 11-15 novembro 2006, Embrapa Informática Agropecuária/INPE, p.145-154.

VALERIANO, M. M.; KUPLICH, T. M.; STORINO, M.; AMARAL, B. D.; MENDES JÚNIOR., J. N.; Lima, D. Modeling small watersheds in Brazilian Amazônia with SRTM-90m data. Computers \& Geosciences, v.32, n.8, p.1169-1181, 2006.

VALERIANO, M. de M.; ABDON, M. de M. APLICAÇÃO DE DADOS SRTM A ESTUDOS DO PANTANAL. Revista Brasileira de Cartografia, n. 59/01, Abril, 2007. (ISSN1808-0936).

VALERIANO, M. de M. Modelo digital de variáveis morfométricas com dados SRTM para o território nacional: o projeto TOPODATA. Anais... XII Simpósio Brasileiro de Sensoriamento Remoto, Goiânia, Brasil, 16-21 abril 2005, INPE, p. 3595-3602.

VIEIRA, S.R. Geoestatística em estudos de variabilidade espacial do solo. In: Tópicos em ciência do solo, Viçosa, Sociedade Brasileira de Ciência do Solo, 2000. v.1, p.1-54.

Geografia Ensino \& Pesquisa, v. 17, n.3 p. $143-152$, set./dez. 2013

Brito, C. R.; Cardoso, C. R.; Santos, A. S.; Santos, G. R.; Medeiros, N. G.

ISSN 2236-4994 151 


\section{Correspondência:}

Carolina Ramalho Brito - Universidade Federal de Viçosa (UFV). Av. PH Rolfs, s/n. Viçosa, MG Brasil, CEP 36570000.

E-mail: carolrambri@hotmail.com

Recebido em 13 de fevereiro de 2013.

Revisado pelo autor em 05 dezembro de 2013.

Aceito para publicação em 23 de dezembro de 2013. 